\title{
Comparative pharmacovigilance assessment of mortality with pimavanserin in Parkinson disease-related psychosis
}

\author{
Joshua D Brown, PharmD, PhD; Brian Cicali, MS; Carl Henriksen, MS; Irene Malaty, MD; Michael S Okun, MD; \\ and Melissa J Armstrong, MD
}

\section{What is already known about this subject}

- Pimavanserin, compared with placebo in clinical trials, had an increased risk of death ( $4.5 \%$ vs $2.6 \%)$.

- Pimavanserin received FDA approval in April 2016 as a breakthrough therapy, given that the risk of death was comparable with other atypical antipsychotics used in older adults.

- In April 2018, consumer safety groups and media reports raised concerns based on the absolute numbers of death reports in individuals receiving pimavanserin but provided no relevant comparisons for perspective.

\section{What this study adds}

- This study compared pimavanserin with treatment alternatives (eg, clozapine and quetiapine) and other atypical antipsychotics within a population of people with Parkinson disease (PD).

- This study found that pimavanserin had no unforeseen safety signal before media reports and suggested a possible reporting bias after these stories became public knowledge.

- Indirectly compared with treatment alternatives, pimavanserin did not appear to have excess mortality reports when compared with other atypical antipsychotics used in the PD population.

\section{ABSTRACT}

BACKGROUND: Pimavanserin is approved for treatment of Parkinson disease (PD)-related psychosis, but its use has been associated with an increased risk of death during clinical trials, as well as during postmarketing surveillance. Previous reports on the association between pimavanserin and mortality have not taken into account limitations of data sources nor included comparable populations or comparisons to relevant treatment alternatives.
OBJECTIVE: To conduct a comparative pharmacovigilance assessment of pimavanserin vs treatment alternatives and by restricting surveillance data to more representative populations.

METHODS: This was a retrospective analysis of adverse event case reports submitted to the FDA's Adverse Event Reporting System (FAERS) from 2016 through 2019 quarter 3 (Q3). FAERS data are collected from the full population, were further restricted to only those with PD, and were based on PD

\section{Author affiliations \\ Joshua D Brown, PharmD, PhD, and Carl Henriksen, MS, Center for Drug Evaluation \& Safety, Department of Pharmaceutical Outcomes \& Policy, University of Florida College of Pharmacy, Gainesville. Brian Cicali, MS, Center for Pharmacometrics \& Systems Pharmacology, Department of Pharmaceutics, University of Florida College of Pharmacy, Orlando. Irene Malaty, MD; Michael S Okun, MD; and Melissa J Armstrong, MD, Norman Fixel Institute for Neurological Diseases, Department of Neurology, University of Florida, Gainesville.}

AUTHOR CORRESPONDENCE: Joshua D Brown, 352.294.8593; joshua.brown@ufl.edu

J Manag Care Spec Pharm. 2021;27(6):785-90

Copyright $\odot 2021$, Academy of Managed Care Pharmacy. All rights reserved.

medication use. Reports were assessed for exposure to pimavanserin, clozapine, quetiapine, haloperidol, and other atypical antipsychotics. The outcome of interest was all-cause death. A proportional reporting ratio (PRR) and $95 \%$ confidence limits were calculated for each 2 by 2 contingency of outcome (death) and exposure (pimavanserin and others). For each outcome/exposure pair, the baseline population was altered to include the full FAERS sample, only reports with $P D$, reports with PD treated with 
levodopa, and reports with PD treated with multiple PD medications. The sample was also stratified by time period before April 2018 and after September 2018 to capture periods of public knowledge and federal response. A lower $95 \% \mathrm{Cl}$ (Lower95Cl) $\geq 2$ for the PRR was considered as the accepted threshold for a drug safety signal.

RESULTS: As of 2019 Q3, there were 2,287 reports of death associated with pimavanserin. Compared within the full FAERS base population, pimavanserin yielded a PRR Lower $95 \mathrm{Cl}=2.08$ but was smaller when restricted to comparison among only a base population with $\mathrm{PD}$ (Lower95Cl=1.09), PD treated with levodopa (Lower95Cl=1.15), or PD treated with multiple PD medications (Lower $95 \mathrm{Cl}=1.63$ ). Metrics for quetiapine, clozapine, and other atypical antipsychotics were similar in magnitude. Stratification by time showed a possible reporting bias associated with pimavanserin, since no safety signal was detected before April 2018; however, a signal was present thereafter.

CONCLUSIONS: Compared in context with treatment alternatives for patients with $\mathrm{PD}$, pimavanserin was not associated with excess reports of death in the FAERS data. This information should be used in shared decision making between physicians and PD patients to balance the risks and benefits of pimavanserin and other treatments for PD psychosis.

Pimavanserin is the only drug approved for psychosis related to Parkinson disease (PD). ${ }^{1}$ Pimavanserin lacks the dopaminergic, adrenergic, histaminergic, or muscarinic affinity evident in existing antipsychotic therapies ${ }^{2}$ and likely provides a different side effect profile compared with other antipsychotics. Importantly, pimavanserin did not worsen PD symptoms (as is common with many other antipsychotics) in the randomized controlled trial leading to its approval. ${ }^{1}$ Similar to other antipsychotics, pimavanserin prescribing information includes a black box warning for an increased risk of death based on the risk assessment by the US Food and Drug Administration's (FDA) that compared pimavanserin with placebo (mortality risk over 10 weeks, $4.5 \%$ vs $2.6 \%)^{3}$

By April 2018, several lay media reports revealed potential concerns related to pimavanserin that had been highlighted by consumer and drug safety groups. ${ }^{4,5}$ These reports cited an increasing number of death reports submitted to the FDA for pimavanserin and reported these numbers as higher than any other drug treatment alternatives during this time period. In September 2018, the FDA concluded there was not a need for additional warnings or withdrawal from the market. ${ }^{6}$

The ongoing debate regarding the safety of pimavanserin has important implications for individuals with PD. Psychosis symptoms include hallucinations, delusions, and psychotic phenomenon, and prevalence has been reported to be as high as $60 \% .^{7}$ PD psychosis increases with disease duration and tends to worsen over time. ${ }^{7,8}$ Studies show that PD psychosis is associated with increased caregiver burden, ${ }^{9}$ increased nursing home placement ${ }^{10}$ and higher risk of death. ${ }^{11}$ Thus, effective treatments for PD psychosis have potentially important implications for individuals with PD, for families, and for the health care system inclusive of payers.

Effective treatments for PD psychosis are scarce. The Movement Disorders Society considers clozapine and pimavanserin as "clinically useful" and quetiapine as "possibly useful." Other antipsychotics are labeled with "unacceptable risk" due to risks of worsening parkinsonism and increased mortality. ${ }^{12}$ Clozapine is rarely used in clinical practice due to the need for frequent blood monitoring and registration in a Risk Evaluation and Mitigation Strategy program. Quetiapine is frequently used off-label for PD psychosis but is associated with an increased mortality risk in PD..$^{13}$ Particularly in the context of severe psychosis, individuals with $\mathrm{PD}$ and their families require reliable information regarding treatment risks to accurately weigh potential benefits and harms of psychosis therapies.

Data used for postmarketing surveillance, the FDA's Adverse Event Reporting System (FAERS), provide a robust means to capture potential drug safety concerns but can lead to inaccurate conclusions, especially if taken out of context. ${ }^{14}$ These data are particularly sensitive to public knowledge of potential drug safety issues. Increased reporting has been noted with newly marketed, branded medications, and it is known that increased reporting can lead to possible reporting biases. ${ }^{15}$

Given limitations in previous reports and media coverage that relied on the raw counts of death reports, this study compared the risk of death associated with pimavanserin using a comparative pharmacovigilance approach. This approach compared pimavanserin in relative terms with other standard treatment alternatives for psychosis in PD. Possible reporting bias was accounted for using the spontaneous adverse reporting systems. Finally, we sought to control biases introduced when comparing specialized patient populations with the overall population represented in the data resource.

\section{Methods}

FAERS data from 2016 through 2019 quarter 3 (Q3) were used. FAERS captures information on outcomes, suspected medications, concomitant medications, and patient demographic information. Each FAERS report was flagged 


\section{FIGURE 1 Proportional Reporting Ratio for Death Among Pimavanserin Users Compared With Different Baseline Populations and Time Periods Before and After Public Awareness of Drug Safety}

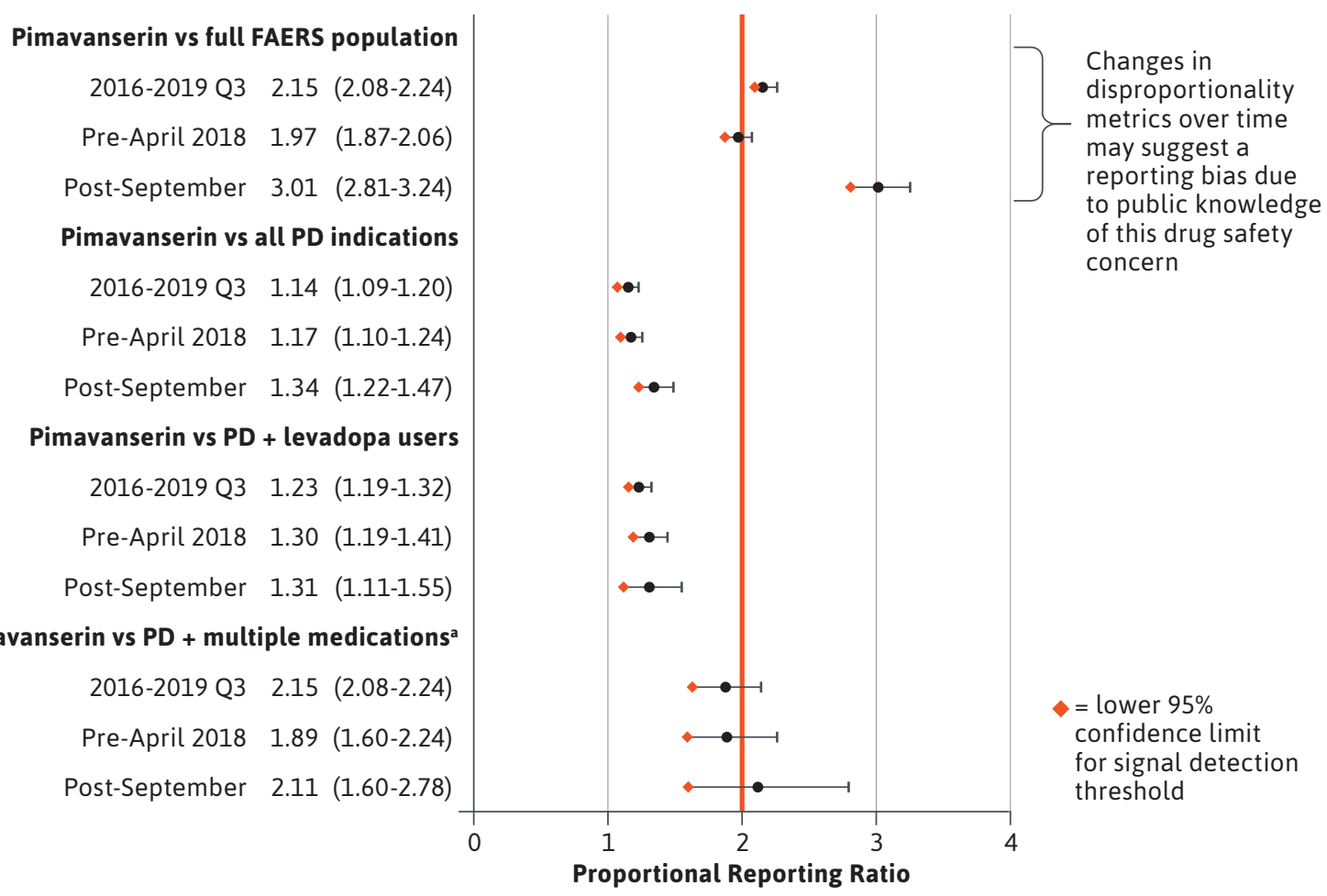

Note: Red diamonds indicate the lower $95 \%$ confidence interval (Lower $95 \mathrm{Cl}$ ) of the $95 \%$ confidence interval. Lower $95 \mathrm{Cl} \geq 2$ (red vertical line) is commonly accepted as a signal detection metric in pharmacovigilance, which would indicate an imbalance of an outcome associated with a particular treatment. The analysis included the full time period 2016 through 2019 Q3 and was also stratified by the first media coverage (April 2018) and after the FDA's reiteration of no additional actions needed (September 2018).

aMultiple PD medications included dopamine agonists, COMT inhibitors, and other PD-specific treatments. Could be any combination of 2 or more PD-related treatments.

$F D A=U S$ Food and Drug Administration; $P D=$ Parkinson disease; $Q 3=$ quarter 3.

based on medications reported that included pimavanserin; levodopa; levodopa plus at least 1 other PD medication (ie, COMT inhibitor, dopamine agonist, or MAO-B inhibitor); quetiapine; clozapine; haloperidol; or other antipsychotics. Quetiapine and clozapine were selected as alternative antipsychotics for PD-related psychosis, whereas haloperidol and a composite group of the other antipsychotics (olanzapine, aripiprazole, ziprasidone, paliperidone, asenapine, lurasidone, iloperidone, cariprazine, and brexipiprazole) were included for additional comparisons, although they are not typically used for PD-related psychosis.

To provide comparative pharmacovigilance metrics and more homogenous patient populations, we varied the base population by medication use to move from the overall population (the full FAERS sample) to a population of users with PD based on use of PD-related medications with listed indications on the FAERS report, including "Parkinson's disease," "Parkinson's disease psychosis," or "parkinsonian rest tremor." We divided the PD group further based on those who used levodopa only or who used levodopa in addition to 1 or more other PD associated medications to possibly differentiate among those with different stages or severity of PD. By doing so, rather than comparing pimavanserin with all other medications in the full population, the comparisons created by this design evaluate pimavanserin indirectly with other antipsychotics nested within subpopulations with confirmed PD. We further stratified by time period and included only reports before April 2018 (the first major media story) and after September 2018 (after FDA statement). 
We analyzed 2 by 2 tables by antipsychotic medication exposure (pimavanserin, quetiapine, clozapine, haloperidol, and other antipsychotics) and outcome (mortality) for each of the 3 populations identified (full FAERS sample, PD using levodopa only, PD using levodopa+other PD medications; Supplementary Figure 1, available in online article). A proportional reporting ratio (PRR) with $95 \%$ CI was calculated. ${ }^{16}$ PRR measures the imbalance of reported events in the underlying population (Figure 1). ${ }^{16}$ Commonly accepted approaches in pharmacovigilance generally recognize a lower confidence limit (Lower95CI) $\geq 2.0$ as the threshold to indicate a potential safety signal that might initiate a cascade of further investigation to determine if regulatory action is needed. ${ }^{16}$ PRR metrics answer the following question: "Is the outcome [death] experienced more than expected in users of the exposure [pimavanserin]." As the base population changes, it more specifically answers the question within that specific group, ie, "Is the outcome [death] experienced more than expected in users of the exposure [pimavanserin] in a population of patients with PD using levodopa." All analyses were conducted using SAS version 9.4 (SAS Institute).

\section{Results}

As of 2019 Q3 ( 3.5 years of drug availability), there were 2,287 reports of death associated with pimavanserin out of over 4.3 million FAERS case reports and 11,203 total pimavanserin reports. The overall FAERS population had a mean age of 56 (median 60) years; nearly $54 \%$ were female sex; and nearly $73 \%$ of reports were from the United States. Among those with PD indications or pimavanserin use on the case report, mean ages were higher (age 73 and 76 years), included more male sex (57.5\% and $61.4 \%)$, and varied in the country source of the report (61.9\% and nearly $100 \%$ from the United States). Demographics of the case reports are shown in Supplementary Table 1 (available in online article).

In the full FAERS base population and the full data time period, pimavanserin use yielded a PRR Lower95CI $=2.08$ compared with no use (Figure 1). Restricting to the population of PD patients (Lower95CI=1.09), PD patients using levodopa (Lower95CI=1.15), and PD patients using multiple PD medications (Lower95CI=1.63) diminished the safety signal for pimavanserin (Figure 1). Stratification by time showed a possible reporting bias associated with pimavanserin, since the PRR was below the prespecified threshold (Lower95CI =1.87) before the April 2018 media attention and increased after September 2018 (Lower95CI=2.81), despite FDA assertion of no safety issue. Other antipsychotics were not affected by this time stratification.

\section{TABLE 1 Comparative PRRs for Treatment Alternatives and Other Antipsychotics and Association With Mortality Reports in FAERS 2016-2019 Q3 in the Overall FAERS Population, in Only Those With PD, in Those With PD Only Using Levodopa, and in Those With PD Using Levodopa Plus One Other PD Medication}

\begin{tabular}{|c|c|}
\hline Baseline population & $\operatorname{PRR}(95 \% \mathrm{Cl})$ \\
\hline \multicolumn{2}{|l|}{ Pimavanserin } \\
\hline Full FAERS population & $2.15(2.08-2.24)$ \\
\hline Parkinson disease & $1.14(1.09-1.20)$ \\
\hline +Levodopa users & $1.23(1.15-1.32)$ \\
\hline +Multiple PD medications & $1.86(1.63-2.12)$ \\
\hline \multicolumn{2}{|l|}{ Quetiapine } \\
\hline Full FAERS population & $1.76(1.72-1.81)$ \\
\hline Parkinson disease & $1.29(1.20-1.39)$ \\
\hline +Levodopa users & $1.36(1.25-1.48)$ \\
\hline +Multiple PD medications & $1.95(1.65-2.29)$ \\
\hline \multicolumn{2}{|l|}{ Clozapine } \\
\hline Full FAERS population & $1.82(1.77-1.88)$ \\
\hline Parkinson disease & $1.50(1.33-1.69)$ \\
\hline +Levodopa users & $1.53 \quad(1.35-1.73)$ \\
\hline +Multiple PD medications & $1.82(1.37-2.42)$ \\
\hline \multicolumn{2}{|l|}{ Haloperidol } \\
\hline Full FAERS population & $1.59(1.51-1.67)$ \\
\hline Parkinson disease & $1.74(1.20-2.50)$ \\
\hline +Levodopa users & $2.35(1.53-3.61)$ \\
\hline +Multiple PD medications & $2.48(1.16-5.31)$ \\
\hline \multicolumn{2}{|l|}{ Other atypical antipsychotics } \\
\hline Full FAERS population & $1.04(1.01-1.05)$ \\
\hline Parkinson disease & $1.26(1.17-1.36)$ \\
\hline +Levodopa users & $1.34(1.24-1.45)$ \\
\hline +Multiple PD medications & $1.97(1.68-2.30)$ \\
\hline
\end{tabular}

Quetiapine in the base population (Lower95CI=1.72), in the PD only population (Lower95CI=1.20), PD+levodopa (Lower95CI $=1.25)$, and $\mathrm{PD}+$ multiple medications (Lower95CI $=1.65$ ) also showed no strong signals and were similar in magnitude compared with pimavanserin in the PD restricted groups (Table 1). Similarly, clozapine in the base population (Lower95CI=1.77), in the PD only 
group (Lower95CI=1.33), PD+levodopa (Lower95CI=1.35), and PD+multiple medications (Lower95CI $=1.37$ ) did not reveal strong signals. Haloperidol also had no signals in these 4 populations (Lower95CI=1.51, 1.20, 1.53, 1.16, respectively). The other atypical antipsychotics showed no strong signals (Table 1).

\section{Discussion}

This analysis revealed that a pharmacovigilance signal associated with pimavanserin and death diminished when the underlying population was comparable with the treated population (ie, those with PD medication use). Further, time stratification based on secular trends of the media reports and regulator statements revealed a possible reporting bias associated with public knowledge of these reports. This scenario likely increased submissions to FAERS regarding pimavanserin. While no direct comparisons can be made among those with PD psychosis using pimavanserin, clozapine, or quetiapine, the similar results for each group indirectly suggest similar mortality risk among the atypical antipsychotics currently used for PD psychosis.

Individuals with PD requiring psychosis treatment are likely to have a higher risk of death when compared with the full FAERS population, regardless of antipsychotic drug use. Individuals with PD were much older and were predictably more male than those within the general FAERS population. Further, individuals with $\mathrm{PD}$ psychosis requiring treatment tend to have a more advanced disease state..$^{12}$ Psychosis is one predictor of mortality, but so are older age (in general and at PD onset), male sex, more severe motor symptoms, and dementia, ${ }_{11}^{11}$ factors that are also associated with a higher risk of psychosis. Selecting a similar control group and the base population when assessing pimavanserin was critical to achieve a more accurate interpretation of its risk profile.

Choosing an appropriate therapy involves shared decision making with individuals with PD and caregivers in order to weigh their priorities, the available evidence, and assessments of risk. Most available antipsychotics work via dopaminergic pathways and can therefore worsen PD motor features such as tremor, rigidity, and bradykinesia. Pimavanserin works on the serotonergic pathway and did not worsen PD motor symptoms in its pivotal clinical trial. ${ }^{1}$ However, all antipsychotics, including pimavanserin, are associated with an increased mortality risk in previous studies of PD populations. ${ }^{3,13}$

It is critical that clinicians discuss the FDA's risk assessment and black box warning for individuals with PD psychosis with each family. ${ }^{3}$ This process facilitates informed decision making. It is also important to accurately interpret pharmacovigilance data, particularly in the context of sensationalized media reporting.

\section{LIMITATIONS}

The analytical framework of our study aimed to control design limitations inherent in the FAERS data. However, this analysis lacked additional clinical variables to adjust for the severity of disease and to control external influences on the reports submitted to the FDA. Importantly, FAERS data lack a denominator reflective of medication use in the population. By restricting the base population by levodopa use with or without other PD medications, we assumed that individuals had PD. This assumption may not have been true for the entire cohort. This could explain why none of the antipsychotics evaluated showed a potential safety signal (Lower95CI) despite previous research suggesting increased mortality in individuals with $\mathrm{PD}$ receiving antipsychotics. Finally, signal detection methods can only identify, but not rule out, potential drug safety issues. Ongoing research is needed to understand the risk of death associated with antipsychotics in the PD patient population.

\section{Conclusions}

Media reports in 2018 likely overestimated the risks associated with pimavanserin use in PD psychosis. This scenario then led to additional overreporting of events, skewing subsequent risk assessments using FAERS data. Our data suggest that there does not appear to be an increased risk of mortality for pimavanserin in PD when compared with use of other atypical antipsychotics within the PD patient population. This report demonstrates how comparative pharmacovigilance analyses can balance measures of Parkinson disease antipsychotic mortality.

\section{DISCLOSURES}

No outside funding supported this study. The authors report no disclosures or conflicts of interest relevant to this study.

Armstrong receives research support from the NIA(P30AG047266, R01AG068128) and the Florida Department of Health (grant 20A08). She is the local principal investigator of a Lewy Body Dementia Association Research Center of Excellence. She also receives compensation from the American Academy of Neurology for work as an evidence-based medicine methodology consultant. She is on the level of evidence editorial board for Neurology and related publications (uncompensated), receives publishing royalties for Parkinson's Disease: Improving Patient Care (Oxford University Press, 2014), and has received an honorarium for presenting for Medscape CME in 2018.

Okun serves as a consultant for the Parkinson's Foundation and has received research grants from NIH, Parkinson's Foundation, the Michael J. Fox Foundation, the Parkinson Alliance, Smallwood 
Foundation, the Bachmann-Strauss Foundation, the Tourette Syndrome Association, and the UF Foundation. Okun has participated as a site principal investigator and/or co-investigator for several $\mathrm{NIH}$-, foundation-, and industry-sponsored trials over the years but has not received honoraria.

Malaty has participated in research funded by the Parkinson Foundation, Tourette Association, Dystonia Coalition, Abbvie, Boston Scientific, Eli Lilly, Neuroderm, Pfizer, Revance, and Teva. She has received travel compensation and/or honoraria from the Tourette Association of America, NeuroChallenge Foundation and $\mathrm{NIH} /$ Neurobiology of Disease in Children, Parkinson Foundation, Medscape, International Association of Parkinsonism and Related Disorders, and Cleveland Clinic, and royalties from Robert Rose publishers. The other authors have no disclosures.

\section{REFERENCES}

1. Cummings J, Isaacson $S$, Mills R, et al. Pimavanserin for patients with Parkinson's disease psychosis: a randomised, placebo-controlled phase 3 trial. Lancet. 2014;383(9916):533-40.

2. Vanover KE, Weiner DM, Makhay M, et al. Pharmacological and behavioral profile of N-(4-fluorophenylmethyl)N-(1-methylpiperidin-4-yl)-

N'-(4-(2-methylpropyloxy)

phenylmethyl) carbamide (2R,3R)-

dihydroxybutanedioate (2:1) (ACP-103), a novel 5-hydroxytryptamine(2A) receptor inverse agonist. J Pharmacol Exp Ther. 2006;317(2):910-918.

3. US Food and Drug Administration. Nuplazid (pimavanserin). 2016. Accessed January 5, 2020. https://www.accessdata.fda.gov/drugsatfda_docs/ nda/2016/207318Orig1s000TOC.cfm
4. Ellis B, Hicken M. FDA worried drug was risky; now reports of deaths spark concern. 2018. Accessed January 2, 2020. https://www.cnn.com/2018/04/09/ health/parkinsons-drug-nuplazid-invs/ index.html

5. Institute for Safe Medical Practices. QuarterWatch $^{\mathrm{TM}}$ (includes data from Quarter 3, 2018) Focus on three psychoactive drugs: gabapentin, pregabalin, and pimavanserin. 2019. Accessed January 5, 2021. https://www.ismp.org/resources/ quarterwatchtm-includes-data-quarter3-2018-focus-three-psychoactive-drugsgabapentin

6. U.S. Food and Drug Administration. FDA analysis finds no new or unexpected safety risks associated with Nuplazid (pimavanserin), a medication to treat the hallucinations and delusions of Parkinson's disease psychosis. 2018. Accessed January 2, 2020. https://www.fda.gov/ drugs/drug-safety-and-availability/ fda-analysis-finds-no-new-or-unexpected-safety-risks-associated-nuplazidpimavanserin-medication.

7. Forsaa EB, Larsen JP, Wentzel-Larsen T, et al. A 12-year population-based study of psychosis in Parkinson disease. Arch Neurol. 2010;67(8):996-1001.

8. Goetz CG, Stebbins GT, Ouyang B. Visual plus nonvisual hallucinations in Parkinson's disease: development and evolution over 10 years. Mov Disord. 2011;26(12):2196-200.

9. Mosley PE, Moodie R, Dissanayaka N. Caregiver burden in parkinson disease: a critical review of recent literature. J Geriatr Psychiatry Neurol. 2017;30(5):235-52.
10. Aarsland D, Larsen JP, Tandberg E, Laake K. Predictors of nursing home placement in Parkinson's disease: a population-based, prospective study. J Am Geriatr Soc. 2000;48(8):938-42.

11. Forsaa EB, Larsen JP, Wentzel-Larsen T, Alves G. What predicts mortality in Parkinson disease? a prospective population-based long-term study. Neurology. 2010;75(14):1270-76.

12. Seppi K, Ray Chaudhuri K, Coelho M, et al. Update on treatments for nonmotor symptoms of Parkinson's disease-an evidence-based medicine review. Mov Disord. 2019;34(2):180-98.

13. Weintraub D, Chiang C, Kim HM, et al. Association of antipsychotic use with mortality risk in patients with parkinson disease. JAMA Neurol. 2016;73(5):535-41.

14. Moore N, Hall G, Sturkenboom M, Mann R, Lagnaoui R, Begaud B. Biases affecting the proportional reporting ratio (PPR) in spontaneous reports pharmacovigilance databases: the example of sertindole. Pharmacoepidemiol Drug Saf. 2003;12(4):271-81.

15. Hoffman KB, Dimbil M, Erdman CB, Tatonetti NP, Overstreet BM. The Weber effect and the United States Food and Drug Administration's Adverse Event Reporting System (FAERS): analysis of sixty-two drugs approved from 2006 to 2010. Drug Saf. 2014;37(4):283-94.

16. Evans SJ, Waller PC, Davis S. Use of proportional reporting ratios (PRRs) for signal generation from spontaneous adverse drug reaction reports. Pharmacoepidemiol Drug Saf. 2001;10(6):483-86. 